\title{
Reflets
}

Revue ontaroise d'intervention sociale et communautaire

\section{Réflexions sur la santé et la précarité du système universel de la santé - Entrevue avec l'Honorable Monique Bégin}

\section{Louise Picard et Richard Carrière}

Volume 5, numéro 2, automne 1999

La santé des francophones de l’Ontario

URI : https://id.erudit.org/iderudit/026268ar

DOI : https://doi.org/10.7202/026268ar

Aller au sommaire du numéro

Éditeur(s)

Reflets : Revue ontaroise d'intervention sociale et communautaire

ISSN

1203-4576 (imprimé)

1712-8498 (numérique)

Découvrir la revue

Citer ce document

Picard, L. \& Carrière, R. (1999). Réflexions sur la santé et la précarité du système universel de la santé - Entrevue avec l'Honorable Monique Bégin. Reflets, 5(2), 22-41. https://doi.org/10.7202/026268ar

Tous droits réservés (C) Reflets : Revue ontaroise d'intervention sociale et communautaire, 1999
Ce document est protégé par la loi sur le droit d'auteur. L’utilisation des services d'Érudit (y compris la reproduction) est assujettie à sa politique d'utilisation que vous pouvez consulter en ligne.

https://apropos.erudit.org/fr/usagers/politique-dutilisation/ 


\section{Réflexions sur la santé et la précarité du système universel de la santé}

M adame M onique Bégin, C.P., O.C., a été à tour de rôle enseignante, ministre fédéral, professeure et administratrice universitaire. Elle est présentement professeure émérite à la Faculté des Sciences de la santé à l'U niversité d'O ttawa. C ette entrevue téléphonique a été réalisée le 22 septembre, 1999 par Louise Picard et $\mathrm{R}$ ichard $\mathrm{C}$ arrière.

R eflets : C omme point de départ pourriez-vous nous donner votre définition de la santé?

M. Bégin: Alors moi, comme définition de la santé, je souscris à celle de l'O rganisation mondiale de la santé qui dit que la santé c'est un état de complet bien-être physique, mental et social et que ce n'est pas seulement l'absence de maladie. $M$ ais si j'ajoute un peu à ma façon ce que ça veut dire ou comment moi je le comprends, c'est que la santé est quand même une notion relative et qu'on est en santé en autant qu'on vit un état d'équilibre entre lesorganes physiologiques et puis les fonctions du corps humain. Alors ça implique de l'équilibre, de l'harmonie entre toutes les possibilités de la personne. Ç a implique une capacité d'adaptation aussi grande que possible aux défis de la vie moderne parce que la santé n'est pas une situation en noir et blanc, elle est un équilibre relatif qui est constamment, si je peux dire, renégocié entre la personne et les défis de son style de vie, puis de son environnement. Alors, et puis j'aurais dû ajouter, dans une absence de douleur ou un minimum de douleur parce qu'une personne peut être relativement en bonne santé mais vivre avec une certaine douleur, en même temps. Je m'excuse de cette longue définition. 
R eflets: $\quad \mathrm{N}$ on, c'est tellement important. C ette vision globale a naturellement un impact sur toutes les questions sur lesqiels on va se pencher cet après-midi. II serait aussi utile pour nos lecteurs si vous pouviez retracer un peu les principales étapes de votre carrière dans le domaine de santé.

M. Bégin : Alorsmoi, je suisune sociologue de formation qui a plutôt travaillé dans des questions de changement social et j'ai été catapultée dans la vie politique. J'ai été, je donne mon titre exact à l'époque là, ministre de la Santé nationale et du Bien-être social. C 'était le gros ministère qui comportait tous les programmes. Je l'ai été de 1977 à 1984. J'y ai pris un goût extraordinaire pour les questions de politique sociale et lesquestions de santé. La vie a fait qu'ensuite, 2 ans après, en devenant la première titulaire de la chai re conjointe U niversité d' $O$ ttaw a et $U$ niversité $C$ arleton en Études desfemmes, je créais les premiers cours Femmes et Santé, et j'ai fait ça pendant quatre ans de 1986 à 1990. En 1990, j'ai été choisie comme doyenne de la nouvelle Faculté des Sciences de la santé de I'U niversité d'O ttawa, qui est la Faculté de toutes les professions en santé à l'exception de la médecine. Pendant que j'étais doyenne et depuis lors, j'ai fait et je continue à faire du bénévolat lourd, $\mathbf{s}$ je peux m'exprimer ainsi, en santé. Par exemple, je viens de terminer de présider pendant quatre ans la $\mathrm{R}$ echerche en cancer du sein du C anada. Q uoi d'autre? Je siège àl'Institut de cardiologie d'O ttawa, I'Institut de R echerche clinique de M ontréal, etc. J'ai aussi coprésidé la Commission royale d'enquête sur l'éducation en 0 ntario et j'ai été membre de la Commission indépendante internationale sur la population et la qualité de la vie. Et là aussi on a parlé santé. J'ai plongé dans des dossiers qui m'ont vraiment toujours passionnée, mais malheureusement dans un sens je n'ai jamais été directement impliquée dans la livraison des soins, puis des services. Je ne l'ai pas vécu de cet angle-là. M ais je parle, j'ai oublié de dire que je parlais aussi, je parle des soins de santé parce que la vie a fait que j'ai acquis des connaissances assez uniques et quej'ai une voix officielle qui me permet de dire ce que beaucoup pense, alors je deviens comme une voix. Alors je ne me prends pas pour une autre, mais je veux utiliser ma voix pour dire les choses que je connais ou que j'ai observées et faire réfléchir les gens ou donner des approches-chocs pour les réveiller. 
R eflets : Vos commentaires sont toujours fort intéressants. $D$ ans votre rôle comme ministre de la Santé, comme doyenne, est-œ qu'il y a certains moments ou accomplissements qui sont plus mémorables pour vous?

M. Bégin : Comme ministre de la Santé, c'est sûr que je vais dire le passage unanime en C hambre des C ommunes en avril 1984 de la Loi de la santé du Canada qui, à toute fin pratique, a eu I'heureux effet de contrôler les tickets modérateurs de certains hôpitaux et les surcharges aux patients par les médecins spécial istes. A lors ça, ç'a été une très longue bataille publique si je peux dire, qui s'est échelonnée sur six ansmais qui a quand même fini, malgré toutes les oppositions, avec un vote unanime de la Chambre des Communes, ce qui est exceptionnel. Pour réussir à gagner cette manche, j'avais comme allié numéro un et comme presque le seul grand allié le public, c'était le monde. Alors je pense que ç'a vraiment permisà la société civile, disons de décider qu'elle voulait garder le système de soins de santé intègre et depuis, même s'il y eut beaucoup d'accrochages et beaucoup d'érosion, c'est encore le public qui est le meilleur chien de garde.

$R$ eflets: 0 ui, on entend encore le même message?

M. Bégin: Comme doyenne, j'ai eu la malchance de frapper les coupures pendant six des sept années de mon décanat, donc j'ai dû gérer la décroissance alors que c'était une Faculté en voie de développement. $M$ aisj'ai essayé de la gérer avec les gens, pas contre les gens. O n n'a jamais coupé de programmes, au contraire, on a même réussi à en obtenir d'autres. C 'est sous mon décanat qu'on a créé le Programme de maîtrise d'audiologie et d'orthophonie, un programme qui est conjoint avec la Laurentienne.

C equej'ai tenté de faire, ç'a été de toujours habiliter mescollègues professeurs, au sens anglais de empowered, les habiliter en leur donnant tous les moyens pour plus de compétences. J'ai été la première doyenne, ici à l'U niversité d'O ttawa, à donner des ordinateurs de la meilleure qualité à tous les professeurs, même ceux qui ne savaient pasce que c'était qu'un ordinateur, me disant un jour ça leur servira. $\mathrm{O}$ a créé des laboratoires pour nos étudiants pour qu'ils se sentent fiers, à la mode, et qu'ils aient leurs outils de travail. Parce qu'il ne faut pas oublier que c'est une 
Faculté qui a une caractéristique unique, elle est toujours, toujours, toujours à la remorque de la médecine, elle est toujours jugée la parente pauvre du système des soins de santé.

R eflets: Si on peut revenir aux questions politiques, selon vous, quelles sont les transformations les plus importantes qui ont eu lieu dans le système de santé canadien dans les dernières cinquante années?

M. Bégin : Pour moi, ç'a été la création même de l'assurance-santé. M oi je n'ai été que celle qui corrigeait des erreurs. La création de I'assurance-santé a été faite en deux étapes, la première en 1957, avec la création de la loi qui établissait la gratuité des hôpitaux, et dix ans plus tard, en 1967, avec la loi qui a créé la gratuité des visites aux médecins, qui a pris en charge le coût des visites aux médecins, qui est la base de ce qui est aujourd'hui notre assurancesanté. Ç $a$, ç'a changé la vie des $C$ anadiens d'une façon incroyable. $O \mathrm{r}$, il y a eu toutes sortes d'autres programmes mis en œuvre au fil desannées par lesgouvernements successifs, maisils sont jusqu'à un certain point, mineurs par rapport à cette prise en charge par l'État du coût extraordinaire qu'est le coût de la maladie. A lors, évidemment, je parle du gouvernement fédéral parce que je ne peux pas parler des gouvernements provinciaux beaucoup. Si je parle d'un gouvernement provincial, je vais singulariser celui du Q uébec qui a créé, dans les années 1970, le réseau des CLSC. C'est un réseau de centres qui n'a pas encore donné toute sa mesure mais au moins c'est un réseau organisé à travers toute la province, ce que les autres, ce que l'O ntario, par exemple, n'a pas encore.

R eflets: $\quad N$ on, c'est vrai, c'est un bon point. E $n$ passant $M$ onique, nous avions pensé vous demander les plus grands changements dans le dernier siède, mais là, on s' est dit qu' on allait un peu trop loin. $0 \mathrm{n}$ a négodé ánquante ans, mais même là...

M. Bégin : C'est quand même très intéressant dire cinquante ans. Je vais dire pourquoi. La première fois que le projet d'assurance-santé a été présenté aux $C$ anadiens, c'est quand même il y a à peu près cinquante ans. L'idée est née en 1949 lors d'une conférence fédérale-provinciale qui a suivi la fin de la Deuxième Guerre mondiale et qui s'est appelée la $\mathrm{R}$ econstruction $\mathrm{C}$ onference. C ette conférence proposait, en se basant sur le modèle britannique de 
W illiam Beveridge, le père de l'État-providence, un système complet d'assurance-santé. À l'époque, ça n'a absolument pas pris, si on peut dire, entre les joueurs fédéraux et provinciaux, et s'est revenu par le truchement de la Saskatchewan quand Tommy D ouglas a lui-même créé pour sa province, une assurance-santé que lentement les fédéraux ont ensuite copié et proposé à tout le reste du C anada.

R eflets: L orsque vous étiez ministre de la Santé, vous avez dû lutter pour le maintien du système universel de la santé au $\mathrm{C}$ anada. C omment est-ce que vous expliquez la poussée croissante pour la privatisation de notre système de santé, aujourd'hui?

M. Bégin : J'y vois les mêmes forces en jeu et à l'œuvre et je dirais les mêmes forces sournoisesque cellesauxquellesj'ai dû faire face danslesannées 79 à la fin de 84, c'est-à dire, une section de la médecine organisée, c'est-à-dire, avant tout, une idéologie du secteur privé comme étant le plusefficace, le plusà la fine pointe de la technologie, non seulement le plus efficace dans ses traitements, mais le plus efficient dans son coût. C e qui est une vaste blague et ceux qui soutiennent ce point de vue se retrouvent à peu près de la façon suivante: une section de la médecine organisée et en particulier, certains spécialistes qui se font leurrer par l'appât du gain facile et plus gros que leurs honoraires de médecin; une section du secteur privé de la business, quoi, des gens qui pensent faire des affaires d'or sur le dos des malades et des gouvernementsprovinciaux; et certains gouvernements provinciaux, ça pourrait arriver au fédéral aussi, mais pas en ce moment, maisc'est pas impossible qu'il y en aurait au fédéral aussi, donc, certains gouvernements provinciaux dont la philosophie politique est que le gouvernement existe pour protéger et aider à développer le secteur privé en tout. Je vais prendre un exemple concret. En ce moment, au C anada, c'est clairement la position de la province de l'A lbertacomme gouvernement, au moins elle a l'honnêteté de le dire à haute voix. L'A lberta veut vraiment privatiser une partie de son système de soins de santé, au moinsl'Alberta est plus honnête et c'est la seule qui est franche. $\mathrm{H}$ arris, d'aprèsmoi, a exactement lesmêmes intentions, et la même philosophie et il croit profondément que le secteur privé est meilleur que le secteur public dans le domaine des soins de santé, mais il a quand même assez de savoir-faire politique pour ne pas le 
dire. Alors ça nous donne un résultat que j'appellerais presque hypocrite, parce que ça se passe en coulisseset personne n'a la vérité, n'al'heure juste. Le M anitoba sous la direction de G ary Filmon, qui vient d'être défait, est un autre exemple de gouvernement qui a, par la bande, c'est-à dire en coulisses, a laissé, a aidé à sinstaller, surtout dans les soins à domicile et les soins chroniques, le secteur privé. Alors, c'est des idéologies, des philosophies. C'est comme ça que je pense : les mêmes joueurs sont encore sur la scène, mais ils sont moinset agissent uniquement par en arrière maintenant. Et cesjoueurs se retrouvent, entre parenthèses, dans toutes les provinces. J'ai singularisé troisprovincesqui sont plusfrappantes, maisdesmédecins ou des spécialistes qui veulent faire de la grosse argent plus vite, puis qui se font des cliniques privées, il y en a au Q uébec, il y en a en O ntario. 0 n a su, cet été, par les journaux, qu'il y a au moins 1000 cliniqueset laboratoiresprivésen 0 ntario. 0 r personne, ni le fédéral, ni les citoyens, ni lesprofessionnels de la santé intéressés à la question ne sont capables d'obtenir un inventaire de ce qui existe. C'est tout en sous main, c'est en coulisses que tout ça se passe.

R eflets: Pensez-vous qu'il y a une influence aussi des É tats-U nis?

M. Bégin : $O$ ui, il y a une influence desÉtats-U nis et il y a des tentatives de firmes américaines... II y a une très très forte influence desÉtatsU nis. Et dans son cas, le $C$ anada a une fragilité unique dans les pays industrialisés parce qu'il n'a qu'un voisin et c'est un géant. Alors tout ce qui vient desÉtats- $U$ nis, on pense que c'est magique et que c'est obligatoirement la clé de l'efficacité de gestion et de finance. $C$ 'est complètement faux. Leur système de soins de santé coûte beaucoup, beaucoup, beaucoup plus cher. C'est le plus cher au monde, mais de beaucoup. $\mathrm{N}$ ous sommes le deuxième plus cher au monde, mais eux c'est beaucoup plus et malgré ça, il y a 47 millions d'A méricains qui n'ont aucune assurance-santé, et plusieurs autres millions qui sont sous-assurés. Alors, ils ont une grosse influence chez-nous.

R eflets: M ais tantôt, vous parliez de l'influence du public dans l'appui pour votre projet sur la L oi de la santé. A vec l'écart croissant entre les riches et les pauvres au $\mathrm{C}$ anada aujourd'hui, comment se fait-il que le public semble tomber dans le piège de vouloir aller avec ces discours? 
M. Bégin : $\quad 0$ ui, c'est vraiment très bien dit, tomber dans le piège, parce que c'est un piège qu'on leur tend et ils y tombent. Alors moi, je le verrais comme ceci. La médecine a bien vu à ses affaires et a beaucoup publicisé l'importance de la technologie de haute pointe dans les traitements médicaux offerts. Et elle fait, si je peux dire, une publicité extraordinaire, ne serait-ce que par les programmes de télévision, pas juste les programmes d'affaires publiques. A lors, il y a les programmes d'affaires publiques et il y a les soaps, les téléromans de toutes sortes, qui sont en ce moment aussi bien américains, canadiens anglais que canadiens français. C'est plein de programmesqui se passent dans un hôpital, qui moussent, si je peux dire, l'idée de la médecine de haute technologie, de son coût extraordinaire et des miracles de la science médicale, alors, elle vend au public, évidemment, que si quelqu'un tombe malade, la personne veut avoir the best, veut avoir ce remède miracle, cette panoplie de traitements au laser de la dernière sorte, etc., etc. Les gens savent que des surcharges directes par le médecin, c'est interdit, mais tout se passe par la bande, alors, maintenant, c'est plus une surcharge directe par les spécialistes, $c^{\prime}$ est que ton médecin te dit : «Bien, si vous allez à l'hôpital G énéral, par exemple, à 0 ttawa ou si vous allez à I'H ôpital R égional de Sudbury, ça va être trois mois d'attente, mais vous savez, vous pouvez aller dans le C entre d'achat untel, à la C linique médicale demander le docteur untel. $M$ ais vous serez obligé de payer un petit quelque chose ». Et les gens vont payer pour, par exemple, un $\mathrm{M} \mathrm{R} \mathrm{I}$, un test de résonance magnétique, les gens vont payer de 700 à 750 \$ pour un seul examen puis ils vont se trouver chanceux d'avoir un traitement immédiat sans faire la queue. Ils vont se dire : «Ah bien! Après tout, après tout, moi je peux payer alors je devrais le faire de cette façon-là ». Ils tombent dans le piège pensant aider le système des soins de santé. Je n'ai pas d'autre manière de l'expliquer. C'est mon hypothèse sur ce que je discute en ce moment en public, c'est que, au fil des derniers quinze ans, exactement, depuis qu'on n'a plus de ticket modérateur ou de charge directe par lesmédecins aux patients, les attaques contre l'assurance-santé ont été faites entièrement par en arrière, ç'a été un certain nombre de services qui ont été "délistés ", "dé-assurés » par les régimes d'assurance provinciaux, O HIP en O ntario, par exemple. Si tu payes, ça va 
aller plus vite, alors tu vas payer, et toutes sortes d'autres pratiques qui font des érosions... Ah oui! M aintenant, tu payes partout, ce n'était pas comme ça avant, maintenant, tu payes partout. Du moment que le médecin doit signer quelque chose, si t'as besoin d'un examen médical pour un renouvellement de licence pour une personne âgée, pour un futur employeur, n'importe quoi où le médecin doit signer, tu payes. Personne ne payait avant. D onc, I'ensemble de ces érosions, toujours par en arrière, que le patient découvre d'habitude au moment même où il est dans le cabinet de son médecin, fait que les gens ont perdu le sens de leur entitlement. C'est quoi le mot français. En français, si je disais «de leur droit », c'est pas tout à fait pareil. En français, on n'a pas deux mots. En anglais, il y a right et entitlement. Entitlement, ce sont tes droits acquis, c'est ce que je crois qu'on dirait en français, des droits acquis. Alors les gens ont perdu le sens de règles du jeu claires. Avant, tout était gratuit, maintenant c'est plein d'accrochages, tu ne sais plus, alorsc'est plusfacile, pour n'importe quel système, de créer de plus en plus de manquements aux règles du jeu. C'est ça la situation en ce moment et c'est très grave. $C$ 'est très grave parce que ce sont des érosions, puis une érosion, c'est sournois. Tu ne sais jamais quand l'érosion sera telle que l'établissement va s'effondrer?

R eflets: II y a une autre tendance aussi qu' on observe dans les soins de santé ces jours-a, puis c'est une tendance vers une décentralisation. $0 \mathrm{n}$ voit les provinces et les territoires qui exercent certaines pressions au niveau du fédéral pour décentraliser le contrôle, d'une certaine façon, du système de soins. Q u'en pensez-vous?

M. B égin : Alors si on parle de décentralisation, du fédéral par rapport aux provinces, je pense que c'est un mouvement. Les provinces proposent toujours des variations sur un même thème depuis quinze ans. Elles proposent que ce soit les provinces qui, sous un mode de comité, mettraient elles-mêmes en œuvre la Loi de I'assurance-santé du C anada, la Loi de la santé du C anada, la Loi de 84, là. C'est invraisemblable. Premièrement, c'est une loi fédérale, c'est aux fédéraux d'être imputables de leur loi, d'être redevable politiquement et publiquement. Je veux dire, ils ne peuvent pas se laver les mains d'une loi qu'ils ont eux-mêmes 
passée. $M$ ais ce que ça veut dire au point de vue pratique, c'est que, moi, j'ai eu à travailler avec les dix provinces nécessairement, j'ai très très bien vu la dynamique de groupe qui se passe. Les dix provinces, entre elles, mettons que les fédéraux n'y seraient plus, ne sont absolument pas des égales. L'O ntario va mener le bal, imposant ses volontés aux autres. Le Q uébec va faire bande à part. Les deux autres provinces, la Colombie-Britannique et I'Alberta, qui ont une certaine importance, selon qu'elles s'entendent bien avec le gouvernement de l'O ntario du jour, décideront de se joindre à lui ou de faire cavalier seul. Les petites provinces n'intéressent personne, elles se feront faire la passe. Ç $a$, c'est la première dimension d'une dynamique de groupe qui n'est pas du tout entre égaux. Et la deuxième dimension, c'est que jamais une province va dire à une autre province, si elles ont la charge d'administrer la Loi, de mettre en œuvre la Loi de la santé du C anada, jamais les provinces ne vont, entre elles, s'accuser de manquer à la Loi. Ça va être « gratte-moi le dos, je gratterai le tien », elles vont se renvoyer l'ascenseur mutuellement.A lors moi, je ne peux pas du tout voir de bénéfices à cette décentralisation qu'elles demandent. La décentralisation, entre parenthèses, ce n'est pas tout à fait le bon mot parce que les provinces reçoivent des sommes qui ont été réduites, mais qui sont quand même très importantes, par les transferts fédéraux et tout ce qu'elles ont à faire, c'est d'obéir à cinq conditions. La manière dont elles mettent en œuvre la livraison des soins de santé leur revient et leur appartient complètement. $Y$ 'a pas un chat du fédéral qui met, qui se met le nez là-dedans. A lors c'est vraiment pour ne pas avoir à respecter les cinq conditions de base... Par ailleurs, ces conditions sont tellement élémentaires que si on les respecte pas, il n'y a plus de système de soins de santé universel gratuit.

M. Bégin : Puissi on parle maintenant de décentralisation àl'intérieur d'une province vers ses régions. $M$ ettons que I'O ntario voudrait décentraliser et donner le contrôle aux C onseils régionaux de la santé, par exemple. Je ne sais pas ce qu'il faut penser de ça parce que... Q ui, localement, va prendre le contrôle des Conseils? S'il n'y a pas des règles du jeu de base, des conditions de base qu'ils sont obligés de respecter, comme les provinces doivent respecter 
les cinq principes de la Loi de la santé du C anada, s'il n'y a pas des conditions de base qu'ils doivent respecter, ces $C$ onseils ou les élites locales qui vont être nommées ou élues sur ces $C$ onseils, peuvent définir des soins de santé locaux qui vont àl'encontre de l'assurance- santé universelle. Et les mêmes problèmes qu'avec les provinces peuvent se reproduire. Les provinces peuvent faire du tort à l'assurance-santé, si elles n'y croient pas. Si on ne croit pas dans une assurance- santé universelle, les mêmes problèmes peuvent se produire encore plus au niveau local. C'est ça que j'essaie de vous dire.

R eflets : 0 ui, et justement, pour nous, la population francophone...

M.Bégin: Pour la population francophone de l'O ntario qui, pour moi, n'a pas les mêmes caractéristiques socio- démographiques que celles du N ouveau-Brunswick, la décentralisation, ça va être un moins, parce qu'il y a peu de masse critique attachée à une région précise. D onc, ilsn'auront pas facilement le contrôle. Le plus qu'ils peuvent espérer contrôler peut-être, c'est la région à l'est d'O ttawa, non pas 0 ttawa, c'est impossible qu'ils la contrôlent. La région à l'est d'O ttawa, c'est pas beaucoup de population, ça. $D$ ans la région de Sudbury, je ne crois pas que les gens francophones sont dans un rapport de force suffisant. Je suis sûre qu'ils ne sont pas en position de contrôle.

R eflets : Puiş, mêmes'il forme $30 \%$ de la population, ça neveut pas nécessairement dire qu'il y aura $30 \%$ de représentation sur les comités?

M.Bégin : C'est pour ça que je ne vois pas de gains. J'ajouterais une autre considération. Si les provinces, si une province décide de décentraliser, [... ] parce que je ne dis pas que c'est impossible de bien faire une bonne décentralisation, je crois que c'est possible, mais il faut des règles du jeu et personne à date n'en a jamais présentées. Et l'autre condition, c'est qu'il ne faut pas que le M inistère provincial de la santé, dans notre cas, à Q ueen's Park, garde tous les fonctionnaires qu'il a et réétudie chaque décision. Il faut que la décentral isation régionale soit assortie d'un contrôle budgétaire régional; autrement, c'est une blague.

R eflets: A u plan provindal, en 0 ntario, on a tous pas mal nos opinions sur les effets des compressions budgétaires et les fermetures d'hôpitaux effectuées 
par le gouvernement $\mathrm{H}$ arris depuis son entrée au pouvoir, mais ça nous intéresserait beaucoup d'entendre votre perception sur ce qui se passe?

M. Bégin : Ah ça! C'est bon. Par où commencer! Je vais commencer par mon énoncé de l'approche des réformes que j'aurais voulu voir et après ça, on parlera de qui les a faites et comment. Alors, les réformes qu'il fallait faire dans les hôpitaux, à mon avis, n'impliquaient pas le downsizing et la fusion d'institutions, sauf dans certains cas. $C$ e n'était pas ça avant tout qu'il fallait faire. $\mathrm{Ce}$ qu'il fallait faire avant tout c'était de la rationalisation, ce qui voulait dire que, dansune ville ou une région donnée, on repensait à qui faisait quoi et à qui le faisait mieux, etc. $M$ ais on ne fermait pas nécessairement des institutions. II fallait absolument repenser le système. Pour moi, c'est clair : il fallait fermer un certain nombre de lits d'hôpitaux. M aisil fallait en même tempsouvrir desservices de soins à domicile et communautaires beaucoup plus légers et beaucoup moins chers que des hôpitaux pour faire la transition. Alors, il fallait faire le ménage à l'intérieur des institutions hospitalièrespour éviter les dédoublements, comme deux hôpitaux qui font exactement la même chose dans une ville pas très grosse. II fallait ajouter, au sortir des hôpitaux, une série, un éventail de différents services de soins à domicile et communautaires et avant d'entrer à l'hôpital, l'idéal eût été de créer un réseau de cliniques de soins primaires, un peu comme les C LSC au Q uébec. Ça, c'est ce qu'il fallait faire. Q u'est-ce que la province a fait à la place? Premièrement, elle a créé une commission de restructuration qu'elle a mise sous la direction de D uncan Sinclair, qui était doyen de la faculté de médecine à Q ueen's et elle s'est dégagée de toute responsabilité en disant que c'est une commission de spécialistes qui fait la job. Ça, c'est invraisemblable, on ne peut pas se dégager de la responsabilité politique de la santé. La santé sera toujours politique, de même que l'éducation, c'est tout à fait normal. La commission de restructuration avait le pouvoir d'imposer ses décisions aux hôpitaux, mais n'avait aucun pouvoir autre que celui de faire de recommandations lorsqu'il s'agissait de nouveaux services. Alors ils n'ont jamais été développés. Je trouve ça épouvantable et la province n'a, à toute fin pratique, jamais dével oppé les soins de santé primaires et n'a jamais développé les 
soins pour les malades chroniques, les soins à domicile, les services communautaires d'aide et de soins en santé mentale, etc. Alors ç'est ce qui s'est passé en 0 ntario. Ç'a été vécu par les citoyens aussi avec une grande brutalité et tout ça a été mis en œuvre par la province sousprétexte de fai re des épargnesimportantes en santé. $M$ oi, si je regarde la scène locale d'O ttawa ou Sudbury, deux villes où ça s'est passé de façon particulièrement pénible, je veux dire, j'allais dire vicieuse, maisje ne veux pas aller jusque-là, mais ça été des luttes féroces entre institutions. Ici, à 0 ttawa, il va y avoir des cicatrices pour longtemps et tout ça, est supposé avoir mené à des épargnes importantes. O ù sont passées ces épargnes? Elles n'ont jamais été réinvesties dans le système des soins de santé. $M$ ais moi, je ne suismême pas certaine qu'il y a eu des épargnes importantes. C'est encore un point où on n'a pas d'imputabilité, on n'a pas de public accountability, on n'est pas redevable. Alors, on ne sait pas la vraie réalité, on ne connait pas les effets et les conséquences de la restructuration en 0 ntario ou ailleurs.

R eflets : C ' 'est un excellent point. U $n$ autre exemple possiblement de manque de planification est la pénurie de professionnels présentement dans le domaine des soins de la santé. C ompte tenu de votre formation et de votre expérience, vous avez certainement quelques mots à dire à ce sujet.

M. B égin : Alors... Je vais séparer la formation des médecins de la formation des autres professionnels de la santé. L'A ssociation médicale canadienne fait énormément de bruit, en ce moment, pour dénoncer la terrible, la soi- disant terrible, pénurie de médecins et qu'il faille réouvrir complètement sans quota les Facultés de médecine. Je ne suis pas sûre que ce soit vrai. II reste cependant qu'à l'intérieur du nombre des quotas permis pour chacune des facultés de médecine, on devrait parler de celles de l'O ntario, on a cinq facultés de médecine en 0 ntario; elles ont chacune un quota approuvé par la province. À l'intérieur de ces quotas, il y a visiblement un problème de distribution dans les diverses spécialités. Ç a c'est sûr que là il y a un problème. Je ne connais pas assez le domaine pour savoir quelles approches pourraient être mises en œuvre pour pallier ce problème. Évidemment il y a le vieux problème qu'on a partout au $C$ anada, des régions un peu pluséloignées, des régions du N ord. C omment est-ce qu'on garde 
les médecins dans les régions un peu plus éloignées ou isolées, dans toutes les municipalités du $\mathrm{N}$ ord de chacune des provinces? Ça c'est certainement un problème.

$M$ oi, je pense que ce n'est pas avec du vinaigre qu'on attire les mouches et que ça prend des incitatifs, mais pas nécessairement des incitatifs financiers. Par exemple, les médecins seraient très sensibles, surtout les jeunes médecins qu'on veut attirer dans le $\mathrm{N}$ ord, seraient très sensibles à recevoir, payée par leur établissement c'est-à-dire par la province, une semaine par année de perfectionnement ou de formation dans un grand centre à $\mathrm{H}$ arvard, à San Francisco, à Princeton, etc. Et je fais exprès de choisir des grands centres internationaux; c'est énorme pour eux d'être à la fine pointe, de voir ce qui se fait ailleurs, de rencontrer des collègues, d'avoir les stimulants de la discussion, etc. Ça, ça se ferait, mais je ne sais pas pourquoi ce n'est pas fait.

D euxièmement, les autres professionnels de la santé, alors là, la situation est complètement différente parce qu'il n'y a pas un médecin qui a perdu sa job au $\mathrm{C}$ anada durant la restructuration. C eux qui ont perdu leur job, c'est d'abord les infirmières et ensuite, en gros, l'ensemble des autres professionnels de la santé. Les physiothérapeutes, et de façon moindre les ergothérapeutes, et encore une fois, personne n'a de chiffres exacts, mais on le sait en entendant parler nos anciens étudiants, qui ont réussi à s'ouvrir des cliniques privées parce qu'ils perdaient leur job dans les hôpitaux. Et ça marche, parce que ces services-là sont couverts par bon nombre de plans d'assurance des employeurs et des employés. Eux se sont placés et on a moins perdu d'étudiants dans ces deux programmes-là. En audiologie et surtout en orthophonie, de nombreux postes ont été coupés parce que les orthophonistes sont souvent employés par des conseils scolaires. Les conseils scolaires ont coupé toutes ces professions-là comme étant un luxe qu'ils ne pouvaient plus se permettre. A lors ça, c'est épouvantable.Alorsnosjeunesqui sont dansles écoles secondaires, qui lisent les journaux, qui connaissent les nouvelles dix fois plus vite que nous, ne vont pas aller dans des professions quand ils lisent que les coupures des hôpitaux, puis dans les écoles, sont constantes et très graves. Encore la même chose pour les 
infirmières. Les jeunes qui veulent entrer en sciences infirmières que ce soit au collégial ou à l'université, quand elles lisent et qu'elles entendent parler de toutes les fermetures de lit et les restrictions de budget d'hôpitaux ou même les fermetures d'hôpitaux, elles ne vont certainement pas aller en sciences infirmières! A lors les provinces, elles pensent qu'après, on appuie sur un bouton, puis on change la ligne de conduite, puis on dit : « $O$ ui, oui, on veut 10000 infirmières, a dit $M$ adame Elizabeth W itmer. 0 n veut 10000 infirmières». Pour former une infirmière, c'est un minimum de 4 ans. A lors ça fait des trous extraordinaires, çà, dans les cohortes de professionnels.

R eflets: Par rapport à cette question, quelle est votre perception quant au niveau d'engagement de la province envers la formation des intervenants francophones?

M.Bégin: Je crois pouvoir affirmer que le gouvernement $H$ arris n'a pas vraiment honoré ses engagements pour la formation des francophones. Je crois que ce gouvernement n'y croit tout simplement pas. C'était plusclair, les règles du jeu étai ent beaucoup plus claires avant. II y avait aussi des budgets supplémentai res parce qu'il y a un coût supplémentaire à la formation de francophones dans des régions ou dans des situations minoritaires comme celle de l'O ntario.A lors, je pense qu'on peut dire que le gouvernement n'a pas honoré ses engagements. Moi j'ai trouvé qu'il n'avait aucune sensibilité. $O$ ui, il y a quelques francophonesqui se battent, je n'irai pasjusqu'à dire qu'il n'y a pas de francophonesà l'intérieur du gouvernement provincial qui ne se battent pas, parce quej'ai connu desfonctionnaires franco-ontariensqui faisaient tout pour mettre le sujet de l'avant et se battre en faveur de la formation d'intervenants francophones. M ais, il n'y a pas grand monde qui y croit autour d'eux. D u moins, c'est ce que j'ai vu. La formation clinique dans la santé est aussi importante que la formation dans nos universités ou nos collèges. Le nouveau programme spécial de formation en santé, c'est à travers M ontfort et c'est pour tous les francophones hors $Q$ uébec. Ça, c'est de l'argent de Sheila Copps, ministre de Patrimoine $C$ anada, que le gouvernement fédéral a investi au pire de la crise de M ontfort. A lors, ce n'est pas le gouvernement de I'O ntario qui a investi cet argent-là. 
R eflets: Justement, comment est-ce que vous avez interprété la fermeture de $M$ ontfort ou la menace de fermer $M$ ontfort?

M. Bégin: Alors, moi, j'ai trouvé que de la part de la Commission de restructuration, je connais plusieurs de ses membres, mais je n'ai pas accès à de l'information privilégiée, alors je parle en simple citoyenne. J'ai trouvé que c'était une erreur politique et de psychologie sociale et de sociologie extraordinaire. J'ai trouvé ça invraisemblable de manquer de sensibilité et de sens commun à ce point-là. J'ai trouvé ça une erreur majeure dèsle départ. Ensuite, eh bien! Les gens [... ], les positions se sont durcies, puis les gens se sont entêtés. A lors l'affaire est maintenant devant les tribunaux. Je pense que les gens de $M$ ontfort attendent une décision quelque part en octobre. En même temps que cette crise, mais là, je parle vraiment en mon nom personnel, ç'a vraiment blessé, et ce n'était pas nécessaire, la communauté franco-ontarienne, pas juste la communauté locale franco-ontarienne, parce que ç'a pris valeur de symbole, alors c'était comme une attaque contre l'ensemble des Franco- $O$ ntariens. Ç a, je pense que c'est une blessure qui va durer très longtemps et qui va peut-être faire plus de tort que de bien. Je ne voudrais pas donner l'impression que cet événement réveille un passé douloureux uniquement, parce que le système des soins de santé, pendant ce temps-là, il avance, il bouge, puis il a des forces dynamiques qui le changent. Je ne voudrais pas qu'il y ait trop de retour sur le passé. Je m'explique. La crise a fait qu'une somme de 10 millions de dollars a été débloquée pour créer un Institut de formation et de recherche clinique à M ontfort pour toutes les sciences de la santé et entièrement en français. $\mathrm{Ce}$ qui permettraà $M$ ontfort de devenir réellement et complètement un hôpital universitaire. Ç a, j'en suis très contente, c'est excellent et ça leur donne un rôle. Ç a peut lui donner un rôle par rapport aux communautés francophones hors Q uébec et de l'O ntario. $M$ aisil ne faudrait pas pour autant que $M$ ontfort manque le bateau du virage ambulatoire. M ontfort ne pourra jamais devenir l'hôpital de Mount Sinai, c'est une impossibilité totale. Moi, j'ai vécu pendant sept ans, la difficulté incroyable d'attirer desfrancophones, des professionnels francophones comme professeurs dans ma faculté. M ontfort a exactement le même problème. II ne faudrait 
pas qu'il manque le virage ambulatoire, c'est-à-dire faire équipe avec les projetslocaux, tout en respectant le français évidemment, mais faire équipe avec des projets locaux pour amener la santé aux gens en dehors des hôpitaux, c'est-à- dire travailler comme le fait, par exemple, l'ancien hôpital R iverside, qui, comme le dit $D$ avid Levine, est en train d'être transformé en clinique $M$ ayo du nord de la ville. Les gens ne sont pas obligés de faire affaire avec une dizaine de spécialistes différents, puis attendre... Tout ça, ça va être intégré tout ça. D es services comme ça, M ontfort peut développer ça. M ontfort peut être la branche francophone, si je peux dire, du R iverside. II faudrait qu'il reste ouvert à toutes ces nouvelles possibilités.

R eflets: U ne des tendances qu' on voit poindre dans un avenir rapproché, c' est le vieillissement de la population. $L$ a population francophone est généralement plus âgée que le reste de la population en 0 ntario. C ette situation nous préoccupe. Q uel serait, selon vous, l'impact du vieillissement de la population sur le système de la santé et puis est-ce qu' on planifie de façon adéquate?

M.B égin: Alors moi, je voudrais répondre à cette question en prenant l'exemple d'O ttawa, parce que, à 0 ttawa, tout le réseau $d$ 'institutions de Bruyère que dirige $M$ ichel Bilodeau fait un travail extraordinaire. Alors je pars d'un point de vue où il y a quelque chose de bien fait. $M$ ais en général, un très grand nombre de soins et de services requis par une population vieillissante sont des soins et des services qui n'ont pas besoin de grands hôpitaux universitaires avec la haute technologie. II y a énormément de soins et de services aux personnes âgées qui devraient naturellement se donner dans des cliniques communautaires, où des infirmières cliniciennes, les nurse pracicioners, pourraient faire un travail extraordinaire à des coûts beaucoup moindres. C e serait beaucoup mieux que de les envoyer dans les cliniques externes des hôpitaux ou les faire entrer, sous fausse représentation, aux urgences parce qu'il n'y a pas de services dans la communauté. Les personnes âgées en ce moment sont traitées d'abord et avant tout dans les hôpitaux. C'est sûr que ça va coûter de plus en plus cher parce qu'elles sont de plusen plusnombreuses et elles vivent de plus en plus longtemps. M aisce n'est pas là que devraient être 
traitées les personnes âgées. II y a un certain nombre de cas où la personne a besoin d'une chirurgie très sophistiquée et ç'est évident, cela doit se passer à l'hôpital. M ais il y a énormément de soins aux personnes âgées qui devraient se donner dans des cliniques locales communautaires, et elles n'existent pour ainsi dire pas.

R eflets: Q uant aux soins communautaires, on entend beaucoup parler de l'importance de la prévention, de la promotion de la santé et des déterminants de la santé. Est-ce que, selon vous, ces belles paroles se traduisent vraiment par des changements concrets dans le virage des soins de santé?

M. Bégin : $\quad \mathrm{N}$ on, non. $\mathrm{O}$ sait maintenant, et on sait depuis plusieurs années, et nos grand-mères savaient ça, c'est le simple bon sens, que la santé, c'est beaucoup plus que des médecins et des hôpitaux, que c'est un toit ou une maison décente, une job sûre, du moins la plus sûre possible en tout cas, un environnement sain, etc., etc. En pratique, je suis obligée de constater que l'écart entre les riches et les pauvres, et ce n'est pas seulement au $C$ anada, mais au $C$ anada c'est vraiment inacceptable à cause de nos valeurs de société, donc que l'écart s'agrandit de nouveau. M oi, j'ai tenté avec la création du crédit d'impôt pour enfant et la double augmentation des pensions de vieillesse quand j'étais ministre, de réduire cet écart. O n l'avait réduit un peu et il est en train de s'agrandir de nouveau d'année en année. $0 \mathrm{r}$, la pauvreté est un facteur direct de mauvaise santé, al ors je ne vais pas plus loin que ça. J'en conclus qu'on ne met pas nos priorités à la bonne place. En ce moment, tout le monde demande des réductions d'impôt. Je serais la première à aimer avoir des réductions d'impôt, je n'accepteraiscertainement pas des augmentations d'impôt, mais avec l'argent qui existe, avec les surplus qui existent, on devrait augmenter d'une part le crédit d'impôt pour enfant et d'autre part, réviser les niveaux de pension et on devrait s'attaquer évidemment à la question des sans abris. C'est absolument invraisemblable qu'un pays riche comme le nôtre ait des écarts aussi affreux entre ses riches et ses pauvres. Et ça, c'est la santé. A lors les déterminants de la santé, pour l'instant, ce sont de belles paroles. À ce propos, parlons un instant de notre système de gouvernance, avec ses ministères séparés les uns des autres et concurrentiels entre eux. Eh bien, cette gouvernance ne 
favorise pas la reconnaissance, dans les faits, de l'importance des déterminants de la santé autres que biologiques, [car les interactions entre les déterminants sont multiples et appellent des solutions complexes dont aucun des ministèresne détient en propre la clé]. C'est vrai pour la santé dans la définition globale que j'en ai donnée au début de cette entrevue, mais c'est aussi vrai pour le développement intellectuel. O n devrait avoir des formations, on devrait avoir des programmes universels pour la petite enfance, de trois à cinq ans, partout au $C$ anada. Ç $a$, c'est un investissement fondamental en santé. O $\mathrm{r} \mathrm{H}$ arris, en $\mathrm{O}$ ntario, non seulement a ri de cette recommandation qui était la recommandation numéro un du R apport sur l'éducation que nous lui avons donné en 1995, mais son ministre de l'Éducation a défait aussi bien les junior kindergardens que les kindergardens. Alors les gouvernements quelquefoisn'ont aucun sens des priorités de leurs investissements.

R eflets: En condusion, nous vous invitons à partager avec nous votre vision des soins de santé du $21^{\text {e }}$ siède et aussi si vous voulez même..

M.Bégin : Et ça, en deux minutes, hein!

R eflets: M ême plus que ça! Si soudainement vous étiez la ministre de la Santé et vous aviez un beau budget de plusieurs milliards de dollars, quels seraient vos plans pour dépenser cet argent, pour réaliser votre vision et établir des priorités?

M.Bégin : D'accord. Je dois tout de suite dire que je ne vois pas un budget de milliards de dollars parce que ça, ça n'existe plus ces sortes de budget-là. Alors je partirai du point de vue que je ne pourrais pas avoir que de modestes nouveaux argents, mais ça prend des argents nouveaux. II n'y a aucun doute. A lors d'abord le ministre fédéral. Si j'étais le ministre fédéral de la Santé, la première des choses que je ferais, c'est que j'annoncerais aux provinces que j'ai l'intention d'appliquer la Loi de la santé du C anada et je donnerais à mesfonctionnairesl'ordre d'établir immédiatement un inventaire de toutes les cliniques et de tous les laboratoires privés au $C$ anada, qu'on sache où on va, puis de quoi on parle. Et puis, je commencerais les négociations avec les provinces pour développer un programme de $\mathrm{H}$ ome $\mathrm{C}$ are. $\mathrm{M}$ oi, je choisirais $\mathrm{H}$ ome $\mathrm{C}$ are à Pharma $\mathrm{C}$ are. Je pense qu'en pratique un grand nombre de $C$ anadiens se trouvent couverts plus ou moins par les assurances 
des employeurs. Alors je choisirais les soins à domicile comme priorité absolue si j'étais ministre fédéral et je partirais la négociation avec les provinces. Combien d'argent supplémentaire cela prendrait-il, puis quelles devraient être les conditions de base auxquelles tout le monde décide d'adhérer, quitte ensuite à ce que les provinces décident elles-mêmes la façon dont elles entendent livrer lesservices. Si j'étais ministre provincial, je partirais immédiatement en Colombie-Britannique voir comment cette province a réussi à intégrer de façon très très bénéfique pour les patients, tous les types de soins à domicile. Ilsont particulièrement bien réussi. A lors, ce n'est pas que ce n'est pas faisable. Les histoires à succès, il faut aller les voir, puis en tirer des leçons. M aintenant, ça c'est du côté des soins à domicile. Beaucoup plus difficile, m'apparaît-il, est la question de remplacer les paiements à l'acte, le mode de rémunération des médecins doit changer. Le paiement à l'acte veut dire qu'on paie les médecins pour le processus, et non pas pour le résultat. Ça c'est absolument inacceptable. Et l'ensemble des médecins généralistes, je crois, est prêt à passer à d'autres types de rémunération. II faut que le mode de rémunération permette au médecin, surtout au médecin de famille, au médecin généraliste, de prendre le temps nécessaire pour son malade, pour son patient, pour son client. Les spécialistes devraient aussi, tôt ou tard, changer de mode de rémunération. C'est absolument un des plus grands blocages qu' on a à réformer notre système de soins de santé. C'est absolument épouvantable, c'est vraiment assez grave. Je pense que c'est beaucoup plus difficile. En même temps, je ne sais pas très bien comment faire le lien, mais je pense que le ministre provincial de la Santé doit créer un réseau de $C$ entres de santé communautaires. II devrait y avoir un réseau provincial couvrant toute la province et si les médecins ne sont pas intéressés à en faire partie à cause de ce fameux problème du paiement à l'acte, eh bien, on mettra des nurse practicioners en charge. C'est comme ça que je vois ça. Et puis il y aura d'autres professionnels de la santé dans ces centres. II faut qu'il y ait des médecins qui acceptent les références émanant de ces centres, car ça prend évidemment des références à des médecins, mais il faut absolument que les soins de santé primaires se développent. $\mathrm{Ce}$ n'est pas acceptable qu'une jeune maman avec un nouveau bébé 
simplement enrhumé, une jeune maman qui a tout perdu de la sagesse et du savoir-faire de l'ancien temps, donc que cette jeune maman court à l'urgence de l'hôpital. Pour des rhumes de cerveau, des grippes, ce n'est pas à l'hôpital qu'il faut aller pour ça. En ce moment, on encombre les hôpitaux parce qu'on n'a pas de cliniquescommunautaires. Elles ne doivent pas toutes être pareilles, c'est pas obligatoire. II peut y en avoir de toutes sortes avec des méthodes, des modes, des modèles différents, mais ça en prend partout. M oi je parle de cliniques, là, ouvertes 24 heures par jour, sept jours par semaine.

R eflets: E st-ce que vous avez un dernier commentaire à ajouter?

M. Bégin: Je voudrais juste peut-être finir sur une chose. 0 n n'a pas parlé des médecines alternatives. Et si on cherche à comprendre pourquoi les médecines alternatives prennent tant de place chez les gens, que leur popularité augmente constamment, je pense d'abord et avant tout, que c'est parce qu'elles représentent la nonagression. Elles manifestent une forme de respect un peu oubliée, pour dire le moins, dans les hôpitaux. Elles représentent une tendance qui s'élève contre la déshumanisation du système des soins de santé qui est tellement fragmenté et tellement axé versla haute technologie que les patients ou futurs malades figent. D onc, le succès des médecines alternatives tient avant tout au fait que les gens qui les pratiquent sont à l'écoute. Ils offrent ce que les autres n'offrent pas, soit l'écoute. Ç a, c'est très très important.

R eflets: $\quad M$ erd, on doit vous dire que cette heure et un peu plus, passée en votre compagnie, s'est écoulée très très vite. V os propos sont très intéressants. $\mathrm{N}$ ous sommes certains que nos lectrices et lecteurs apprécieront vos commentaires. $\mathrm{N}$ ous aimerions vous remercier au nom de $\mathrm{R}$ eflets. 